\title{
Thermally stable carbon nanofibers functionalized with poly(dimethylsiloxane) for solid-phase microextraction of polycyclic aromatic hydrocarbons prior to GC analysis
}

\begin{abstract}
The authors describe poly(dimethylsiloxane)-coated carbon nanofibers (CNF-PDMS) for solid-phase microextraction of polycyclic aromatic hydrocarbons (PAHs). The fibers were prepared by a solï gel method and are stable at temperatures up to $350{ }^{\circ} \mathrm{C}$, probably due to the chemical bonds between the coating and the fiber surface. The fibers can be re-used up to 180 times. The CNFs enhance the surface area of the coating compared to that of a plain PDMS fiber and, accordingly, provide higher extraction efficiency. Following thermal desorption, the PAHs were quantified by GC with FID detection. Under optimized experimental conditions, the detection limits of the method range from 5 to $20 \mathrm{pg} \mathrm{mLi} 1$, and response is linear in the 0.017 to $100 \mathrm{ng} \mathrm{mL} \overline{1} 1$ range. The repeatability and reproducibility vary between $4.8 \%$ and $8.6 \%$, and between $4.1 \%$ and $10.2 \%$, respectively. The method was successfully utilized for the analysis of PAHs in (spiked) water samples, with satisfactory recoveries in the range of 90.110799 .9 .
\end{abstract}

Keyword: Gas chromatography; Polyacrylonitrile; Preconcentrationlame ionization detection; Scanning electron microscopy; Solï gel; Transmission electron microscopy 